\title{
Two Phase Sampling Exponential Type Estimators for Ratio and Product of two Population Means in the Presence of Non-response
}

\author{
Kamlesh Kumar $^{1 *}$, Mukesh Kumar ${ }^{2}$ \\ ${ }^{1 *}$ Department of Community Medicine, Rajkiya Medical College, Jalaun (Orai), India \\ ${ }^{2}$ Department of Statistics, Central University of South Bihar, Bihar, India \\ *Corresponding Author: kamalbhu03@gmail.com
}

Available online at: www.isroset.org

Received: 28/Oct/2017, Revised: 10/Nov/2017, Accepted: 30/Nov/2017, Published: 31/Dec/2017

\begin{abstract}
In this paper, two phase sampling exponential type estimators for ratio and product of two population means in the presence of non-response have been proposed and the expressions for the mean suare error of the proposed estimators for ratio and product of two population means in case of fixed sample sizes and also in case of fixed cost, are obtained. The expressions for optimum values of sample sizes are obtained in case of fixed cost and also in case of specified variance. The proposed estimators have been found to be more efficient than the relevant estimators for the fixed values of sample sizes, under the specified conditions. The proposed estimators are also more efficient than the relevant estimators in case of the fixed cost and have less total cost in comparison to the cost incurred in case of relevant estimators for the specified variance. Empirical studies have been given in support of the problem under investigation.
\end{abstract}

Keywords-Two phase sampling, Non-response, Mean square error, Study character, Auxiliary characte.

\section{INTRODUCTION}

Estimation of ratio and product of two population means is widely used in the field of agriculture, socio-economics and medical sciences. A lot of research works for estimating the ratio and product of two population means using one auxiliary character, have been done by Singh [1, 2, 3], Shah and Shah [4], Tripathi [5], Singh [6], Singh [7], Birader and Singh [8] and Upadhayay et al.[9].

For estimating the population mean by using sample values sometimes the information on all units selected in the sample is not collected due to the problem of non-response which occurs due to lack of interest, not at home, refusal and lack of the knowledge regarding to the surveys. Hansen and Hurwitz [10] first suggested a technique of sub sampling from nonrespondent to deal with the problem of non-response. Further by using the known population mean of auxiliary character, various ratio, product and regression type estimators for population mean in the presence of non-response have been proposed by Rao [11,12], Khare and Srivastava [13,14], Singh et al. [15], Kumar and Bhougal [16] and Kumar and Kumar [17]. Sometimes the population mean of auxiliary character is not known. In this situation, two phase sampling ratio, product and regression type estimators have been proposed by Khare and Srivastava [18, 19], Singh and Kumar [20] and Khare et al. [21]. The research works for estimating the ratio of two population means using auxiliary characters in the presence of non-response have also been done by Khare and Pandey [22] and Khare and Sinha [23, 24].

In this paper, we have proposed two phase sampling exponential type estimators for ratio and product of two population means in the presence of non-response. The expressions for the mean suare error of the proposed estimators for ratio and product of two population means in case of fixed sample sizes are obtained. The conditions in which proposed estimators are more efficient in comprision to the relevant estimators are obtained. The expressions for the optimum values of sample sizes are also obtained in case of fixed cost and also in case of specified variance. Empirical studies are also given to show the performance of the proposed estimators in comprision to the relevant estimators.

\section{THE ESTIMATORS}

Let $\left(y_{1}, y_{2}\right)$ denote study characters and $x$ denote the auxiliary character having $l$ th values $Y_{i l} ; i=1,2$, $X_{l} ; l=1,2, \ldots, N$ with their population means $\bar{Y}_{1}, \bar{Y}_{2}$ and $\overline{\mathrm{X}}$ respectively. The population is supposed to be divided in $N_{1}$ responding and $N_{2}$ non-responding units such that $N_{1}+N_{2}=N$. By using the Hansen and Hurwitz [10] technique's, a sample of size $n(<N)$ is drawn from the population of size $N$ by simple random sampling without 
replacement (SRSWOR) method of sampling and found that $n_{1}$ units respond and $n_{2}$ units do not respond in a sample of size $n$ for study character $y_{i} ; i=1,2$. Further from $n_{2}$ nonresponding units, a subsample of size $m\left(=n_{2} / k, k>1\right)$ is drawn by using SRSWOR method of sampling and the information on $m$ units is collected by personal interview. By using the Hansen and Hurwitz [10] technique's, the estimator for the population mean $\bar{Y}_{i}$ of study character $y_{i}$ based on $n_{1}+m$ units is defined as follows:

$\bar{y}_{i}^{*}=\frac{n_{1}}{n} \bar{y}_{i(1)}+\frac{n_{2}}{n} \bar{y}_{i(2)}^{\prime}, i=1,2$,

where $\bar{y}_{i(1)}$ and $\bar{y}_{i(2)}^{\prime}$ denote the means of study character $y_{i}$ based on $n_{1}$ and $m$ units respectively.

The mean square error (MSE) of the estimator $\bar{y}_{i}^{*}: i=1,2$ is obtained as:

$\operatorname{MSE}\left(\bar{y}_{i}^{*}\right)=\frac{f}{n} S_{y i}^{2}+\frac{W_{2}(k-1)}{n} S_{y i}^{\prime 2}$,

where $f=1-\frac{n}{N}, W_{2}=\frac{N_{2}}{N},\left(S_{y i}^{2}, S_{y i}^{\prime 2}\right)$ are population mean squares of study character $y_{i}: i=1,2$ for the entire population and for the non responding part of the population. Hence the conventional estimators for the ratio of two population means $R\left(=\bar{Y}_{1} / \bar{Y}_{2}\right)$ and for the product of two population means $P\left(=\bar{Y}_{1} * \bar{Y}_{2}\right)$ are defined as:

$$
\hat{R}=\frac{\bar{y}_{1}^{*}}{\bar{y}_{2}^{*}} \quad \text { and } \quad \hat{P}=\bar{y}_{1}^{*} * \bar{y}_{2}^{*}
$$

When the population mean $\bar{X}$ of auxiliary character $x$ is not known, a first phase sample of size $n^{\prime}(<N)$ is drawn from population of size $N$ by using simple random sampling without replacement (SRSWOR) method of sampling and collects the information on $n^{\prime}$ units for auxiliary character $x$. Further, a second phase sample of size $n\left(<n^{\prime}\right)$ is drawn from first phase sample of size $n^{\prime}$ by using simple random sampling without replacement (SRSWOR) method of sampling and found that $n_{1}$ units are responding and $n_{2}$ units are not responding in a sample of size $n$ for study character $y_{i}$. Again a subsample of size $m\left(=n_{2} / k, k>1\right)$ is drawn from $n_{2}$ responding units and collects the information on $m$ units for study character $y_{i}$ by personal interview.
Now we define the estimator for population mean $\bar{X}$ of auxiliary character $x$ based on $n_{1}+m$ units corresponding to $n_{1}+m$ units of study character $y_{i}$ which is given as:

$$
\bar{x}^{*}=\frac{n_{1}}{n} \bar{x}_{1}+\frac{n_{2}}{n} \bar{x}_{2}^{\prime},
$$

where $\bar{x}_{1}$ and $\bar{x}_{2}$ denote the means of auxiliary character $x$ based on $n_{1}$ and $m$ units corresponding to $n_{1}$ and $m$ units of study character $y_{i}$.

The mean square error (MSE) of the estimator $\bar{x}^{*}$ is obtained as:

$\operatorname{MSE}\left(\bar{x}^{*}\right)=\frac{f}{n} S_{x}^{2}+\frac{W_{2}(k-1)}{n} S_{x}^{\prime 2}$,

where $\left(S_{x}^{2}, S_{x}^{\prime 2}\right)$ are population mean squares of auxiliary character $x$ for the entire population and for the non responding part of the population.

The classical ratio and product estimators are useful only when the linear relationship between study character and auxiliary character is very strong. Sometimes the linear relationship between study character and auxiliary character is not very strong. In this situation, Singh and Vishwakarma [25] proposed the two phase sampling exponential ratio $\left(t_{R}\right)$ and exponential product $\left(t_{P}\right)$ estimators for the population mean which are given as follows:

$t_{R}=\bar{y}_{1} \exp \left(\frac{\bar{x}^{\prime}-\bar{x}}{\bar{x}^{\prime}+\bar{x}}\right)$

and

$t_{P}=\bar{y}_{1} \exp \left(\frac{\bar{x}-\bar{x}^{\prime}}{\bar{x}+\bar{x}^{\prime}}\right)$,

where $\bar{x}=\frac{1}{n} \sum_{l=1}^{n} x_{l}$ and $\bar{x}^{\prime}=\frac{1}{n^{\prime}} \sum_{l=1}^{n^{\prime}} x_{l}$.

Further Tailor et al. [26] and Lakhre [27] studied Singh and Vishwakarma [25] ratio and product type exponential estimators in double sampling for stratification.

Now using two phase sampling exponential product and exponential ratio estimators, we propose conventional two phase sampling exponential type estimators for ratio and product of two population means in the presence of nonresponse which are given as follows:

$t_{T R 1}=\frac{\bar{y}_{1}^{*}}{\bar{y}_{2}^{*}} \exp \left(\frac{\bar{x}^{*}-\bar{x}^{\prime}}{\bar{x}^{*}+\bar{x}^{\prime}}\right)$

and

$t_{T P 1}=\bar{y}_{1}^{*} * \bar{y}_{2}^{*} \exp \left(\frac{\bar{x}^{\prime}-\bar{x}^{*}}{\bar{x}^{\prime}+\bar{x}^{*}}\right)$ 
Using two phase sampling exponential product and exponential ratio estimators, we propose the alternate two phase sampling exponential type estimators for ratio and product of two population means in the presence of nonresponse which are given as follows:

$t_{T R 2}=\frac{\bar{y}_{1}^{*}}{\bar{y}_{2}^{*}} \exp \left(\frac{\bar{x}-\bar{x}^{\prime}}{\bar{x}+\bar{x}^{\prime}}\right)$

and

$$
t_{T P 2}=\bar{y}_{1}^{*} * \bar{y}_{2}^{*} \exp \left(\frac{\bar{x}^{\prime}-\bar{x}}{\bar{x}^{\prime}+\bar{x}}\right)
$$

\section{THE EXPRESSIONS FOR THE MEAN SQUARE ERROR (MSE) OF THE PROPOSED ESTIMATORS}

In order to derive the expressions for the mean square error of the proposed estimators.

Le $\bar{y}_{1}^{*}=\bar{Y}_{1}\left(1+\varepsilon_{0}\right), \bar{y}_{2}^{*}=\bar{Y}_{2}\left(1+\varepsilon_{1}\right), \quad \bar{x}^{*}=\bar{X}\left(1+\varepsilon_{2}\right)$, $\bar{x}=\bar{X}\left(1+\varepsilon_{3}\right), \bar{x}^{\prime}=\bar{X}\left(1+\varepsilon_{4}\right) \quad$ Such that

$E\left(\varepsilon_{0}\right)=E\left(\varepsilon_{1}\right)=E\left(\varepsilon_{2}\right)=E\left(\varepsilon_{3}\right)=E\left(\varepsilon_{4}\right)=0$.

By using simple random sampling without replacement method of sampling, we have

$E\left(\varepsilon_{0}^{2}\right)=\frac{1}{\bar{Y}_{1}^{2}} V\left(\bar{y}_{1}^{*}\right)=\frac{1}{\bar{Y}_{1}^{2}}\left\{\frac{f}{n} S_{y 1}^{2}+\frac{W_{2}(k-1)}{n} S_{y 1}^{\prime 2}\right\}$,

$E\left(\varepsilon_{1}^{2}\right)=\frac{1}{\bar{Y}_{2}^{2}} V\left(\bar{y}_{2}^{*}\right)=\frac{1}{\bar{Y}_{2}^{2}}\left\{\frac{f}{n} S_{y 2}^{2}+\frac{W_{2}(k-1)}{n} S_{y 2}^{\prime 2}\right\}$,

$E\left(\varepsilon_{2}^{2}\right)=\frac{1}{\bar{X}^{2}} V\left(\bar{x}^{*}\right)=\frac{1}{\bar{X}^{2}}\left\{\frac{f}{n} S_{x}^{2}+\frac{W_{2}(k-1)}{n} S_{x}^{\prime 2}\right\}$,

$E\left(\varepsilon_{3}^{2}\right)=\frac{1}{\bar{X}^{2}} V(\bar{x})=\frac{1}{\bar{X}^{2}} \frac{f}{n} S_{x}^{2}$,

$E\left(\varepsilon_{4}^{2}\right)=\frac{1}{\bar{X}^{2}} V\left(\bar{x}^{\prime}\right)=\frac{1}{\bar{X}^{2}} \frac{f^{\prime}}{n^{\prime}} S_{x}^{2}$,

$E\left(\varepsilon_{0} \varepsilon_{1}\right)=\frac{1}{\bar{Y}_{1} \cdot \bar{Y}_{2}} \operatorname{Cov}\left(\bar{y}_{1}^{*}, \bar{y}_{2}^{*}\right)$ $=\frac{1}{\bar{Y}_{1} \bar{Y}_{2}}\left\{\frac{f}{n} S_{y 1 y 2}+\frac{W_{2}(k-1)}{n} S_{y 1 y 2}^{\prime}\right\}$,

$E\left(\varepsilon_{0} \varepsilon_{2}\right)=\frac{1}{\bar{Y}_{1} \cdot \bar{X}} \operatorname{Cov}\left(\bar{y}_{1}^{*}, \bar{x}^{*}\right)$

$$
=\frac{1}{\overline{Y_{1}} \bar{X}}\left\{\frac{f}{n} S_{y 1 x}+\frac{W_{2}(k-1)}{n} S_{y 1 x}^{\prime}\right\} \text {, }
$$

$E\left(\varepsilon_{1} \varepsilon_{2}\right)=\frac{1}{\bar{Y}_{2} \cdot \bar{X}} \operatorname{Cov}\left(\bar{y}_{2}^{*}, \bar{x}^{*}\right)$

$$
=\frac{1}{\bar{Y}_{2} \bar{X}}\left\{\frac{f}{n} S_{y 2 x}+\frac{W_{2}(k-1)}{n} S_{y 2 x}^{\prime}\right\},
$$

$E\left(\varepsilon_{0} \varepsilon_{3}\right)=\frac{1}{\bar{Y}_{1} \cdot \bar{X}} \operatorname{Cov}\left(\bar{y}_{1}, \bar{x}\right) \quad=\frac{1}{\bar{Y}_{1} \bar{X}} \frac{f}{n} S_{y 1 x}$,

$E\left(\varepsilon_{1} \varepsilon_{3}\right)=\frac{1}{\bar{Y}_{2} \cdot \bar{X}} \operatorname{Cov}\left(\bar{y}_{2}, \bar{x}\right)=\frac{1}{\bar{Y}_{2} \bar{X}} \frac{f}{n} S_{y 2 x}$,

$E\left(\varepsilon_{0} \varepsilon_{4}\right)=\frac{1}{\bar{Y}_{1} \cdot \bar{X}} \operatorname{Cov}\left(\bar{y}_{1}, \bar{x}^{\prime}\right)=\frac{1}{\bar{Y}} \bar{X} \frac{f^{\prime}}{n^{\prime}} S_{y 1 x}$,

$E\left(\varepsilon_{1} \varepsilon_{4}\right)=\frac{1}{\bar{Y}_{2} \cdot \bar{X}} \operatorname{Cov}\left(\bar{y}_{2}, \bar{x}^{\prime}\right)=\frac{1}{\bar{Y}_{2} \bar{X}} \frac{f^{\prime}}{n^{\prime}} S_{y 2 x}$,

$E\left(\varepsilon_{2} \varepsilon_{4}\right)=\frac{1}{\bar{X} \cdot \bar{X}} \operatorname{Cov}\left(\bar{x}^{*}, \bar{x}^{\prime}\right)=\frac{1}{\bar{X}^{2}} \frac{f^{\prime}}{n^{\prime}} S_{x}^{2}$,

$E\left(\varepsilon_{3} \varepsilon_{4}\right)=\frac{1}{\bar{X} \cdot \bar{X}} \operatorname{Cov}\left(\bar{x}, \bar{x}^{\prime}\right)=\frac{1}{\bar{X}^{2}} \frac{f^{\prime}}{n^{\prime}} S_{x}^{2}$,

where $f^{\prime}=1-\frac{n^{\prime}}{N}$.

Using the large sample approximations, the expressions for the mean square error of the proposed estimators $t_{T R 1}, t_{T P 1}$, $t_{T R 2}$ and $t_{T P 2}$ up to the terms of order $(1 / n)$ are given as follows:

$$
\begin{aligned}
\operatorname{MSE}\left(t_{T R 1}\right)= & \operatorname{MSE}(\hat{R})+R^{2}\left[A_{1}\left\{\frac{1}{4} C_{x}^{2}+\left(C_{y 1 x}-C_{y 2 x}\right)\right\}\right. \\
& \left.+A_{2}\left\{\frac{1}{4} C_{x}^{\prime 2}+\left(C_{y 1 x}^{\prime}-C_{y 2 x}^{\prime}\right)\right\}\right], \\
\operatorname{MSE}\left(t_{T P 1}\right)= & \operatorname{MSE}(\hat{P})+P^{2}\left[A_{1}\left\{\frac{1}{4} C_{x}^{2}-\left(C_{y 1 x}+C_{y 2 x}\right)\right\}\right. \\
& \left.+A_{2}\left\{\frac{1}{4} C_{x}^{\prime 2}-\left(C_{y 1 x}^{\prime}+C_{y 2 x}^{\prime}\right)\right\}\right], \\
\operatorname{MSE}\left(t_{T R 2}\right)= & \operatorname{MSE}(\hat{R})+R^{2}\left[A_{1}\left\{\frac{1}{4} C_{x}^{2}+\left(C_{y 1 x}-C_{y 2 x}\right)\right\}\right]
\end{aligned}
$$

and

$$
\operatorname{MSE}\left(t_{T P 2}\right)=\operatorname{MSE}(\hat{P})+P^{2}\left[A_{1}\left\{\frac{1}{4} C_{x}^{2}-\left(C_{y 1 x}+C_{y 2 x}\right)\right\}\right],
$$

where

$\operatorname{MSE}(\hat{R})=R^{2}\left[\frac{f}{n}\left(C_{y 1}^{2}+C_{y 2}^{2}-2 C_{y 1 y 2}\right)\right.$ 


$$
\begin{aligned}
& \left.+\frac{W_{2}(k-1)}{n}\left(C_{y 1}^{\prime 2}+C_{y 2}^{\prime 2}-2 C_{y 1 y 2}^{\prime}\right)\right], \\
\operatorname{MSE}(\hat{P}) & =P^{2}\left[\frac{f}{n}\left(C_{y 1}^{2}+C_{y 2}^{2}+2 C_{y 1 y 2}\right)\right. \\
& \left.+\frac{W_{2}(k-1)}{n}\left(C_{y 1}^{\prime 2}+C_{y 2}^{\prime 2}+2 C_{y 1 y 2}^{\prime}\right)\right],
\end{aligned}
$$

$A_{1}=\frac{1}{n}-\frac{1}{n^{\prime}}, \quad A_{2}=\frac{W_{2}(k-1)}{n}, \quad C_{y 1 y 2}=\frac{S_{y 1 y 2}}{\bar{Y}_{1} \bar{Y}_{2}}$, $C_{y 1 y 2}^{\prime}=\frac{S_{y 1 y 2}^{\prime}}{\bar{Y}_{1} \bar{Y}_{2}}, S_{y 1 y 2}=\rho_{y 1 y 2} S_{y 1} S_{y 2}, S_{y 1 y 2}^{\prime}=\rho_{y 1 y 2}^{\prime} S_{y 1}^{\prime} S_{y 2}^{\prime}$, $C_{y i}=\frac{S_{y i}}{\bar{Y}_{i}}, \quad C_{x}=\frac{S_{x}}{\bar{X}}, \quad C_{y i}^{\prime}=\frac{S_{y i}^{\prime}}{\bar{Y}_{i}}, \quad C_{x}^{\prime}=\frac{S_{x}^{\prime}}{\bar{X}}$, $\left(\rho_{y 1 y 2}, \rho_{y 1 y 2}^{\prime}\right)$ are correlation coefficients between two study characters $y_{1}$ and $y_{2}$ for responding and nonresponding parts of the population and $\left(\rho_{y i x}, \rho_{y i x}^{\prime}\right)$ are the correlation coefficients between $y_{i}(i=1,2)$ and $x$ for the entire population and for the non-responding part of the population.

\section{COMPARISONS OF THE PROPOSED ESTIMATORS WITH RELEVANT ESTIMATORS}

Comparing the proposed estimators $\left(t_{T R 1}, t_{T R 2}\right)$ and $\left(t_{T P 1}\right.$, $t_{T P 2}$ ) with respect to $\hat{R}$ and $\hat{P}$.

$$
\begin{aligned}
\operatorname{MSE}\left(t_{T R 1}\right)<\operatorname{MSE}(\hat{R}) \text { If } \\
\quad\left(\rho_{y 1 x} \frac{C_{y 1}}{C_{x}}-\rho_{y 2 x} \frac{C_{y 2}}{C_{x}}\right)<-\frac{1}{4} \\
\text { and } \quad\left(\rho_{y 1 x}^{\prime} \frac{C_{y 1}^{\prime}}{C_{x}^{\prime}}-\rho_{y 2 x}^{\prime} \frac{C_{y 2}^{\prime}}{C_{x}^{\prime}}\right)<-\frac{1}{4}, \\
\operatorname{MSE}\left(t_{T R 2}\right)<\operatorname{MSE}(\hat{R}) \text { If } \\
\quad\left(\rho_{y 1 x} \frac{C_{y 1}}{C_{x}}-\rho_{y 2 x} \frac{C_{y 2}}{C_{x}}\right)<-\frac{1}{4}
\end{aligned}
$$

and

$$
\begin{aligned}
& \operatorname{MSE}\left(t_{T P 1}\right)<\operatorname{MSE}(\hat{P}) \quad \text { If } \\
& \left(\rho_{y 1 x} \frac{C_{y 1}}{C_{x}}+\rho_{y 2 x} \frac{C_{y 2}}{C_{x}}\right)>\frac{1}{4}
\end{aligned}
$$

and $\quad\left(\rho_{y 1 x}^{\prime} \frac{C_{y 1}^{\prime}}{C_{x}^{\prime}}+\rho_{y 2 x}^{\prime} \frac{C_{y 2}^{\prime}}{C_{x}^{\prime}}\right)>\frac{1}{4}$,

$$
\begin{aligned}
& \operatorname{MSE}\left(t_{T P 2}\right)<\operatorname{MSE}(\hat{P}) \text { If } \\
& \left(\rho_{y 1 x} \frac{C_{y 1}}{C_{x}}+\rho_{y 2 x} \frac{C_{y 2}}{C_{x}}\right)>\frac{1}{4}
\end{aligned}
$$

\section{DETERMINATION OF $n^{\prime}, n$ AND $k$ FOR THE FIXED COST $C \leq C_{0}$}

Let the fixed total cost apart from overhead cost is $C \leq C_{0}$ and the cost function is given by

$$
C^{\prime}=c_{1}^{\prime} n^{\prime}+c_{1} n+c_{2} n_{1}+c_{3} \frac{n_{2}}{k} .
$$

The expected cost $C$ to be incurred on the survey apart from overhead expenses, is given by

$$
C=E\left(C^{\prime}\right)=c_{1}^{\prime} n^{\prime}+n\left(c_{1}+c_{2} W_{1}+c_{3} \frac{W_{2}}{k}\right),
$$

where

$c_{1}^{\prime}-$ the cost per unit of identifying and observing auxiliary character $x$ at the first phase,

$c_{1}$ - the cost per unit of mailing questionnaire visiting the units at the second phase,

$c_{2}$ - the cost per unit of collecting and processing data for the study characters $y_{1}$ and $y_{2}$ obtained from $n_{1}$ responding units,

$c_{3}$ - the cost per unit of obtaining and processing data for the study characters $y_{1}$ and $y_{2}$ (after extra effort) from the sub-sampled units and

$W_{1}=N_{1} / N$ is the response rate in population of size $N$.

The $\operatorname{MSE}(t(j)), j=1,2,3,4$ can be written in term of the notation $V_{0 j}, V_{1 j}, V_{2 j}$ and $V_{3 j}$ which is given as

$$
\operatorname{MSE}(\mathrm{t}(j))=\left[\frac{1}{n} V_{0 j}+\frac{1}{n^{\prime}} V_{1 j}+\frac{k}{n} V_{2 j}-\frac{1}{N} V_{3 j}\right] \text {, }
$$

where $V_{0 j}, V_{1 j}, V_{2 j}$ and $V_{3 j}$ are respectively the coefficients of terms of $n^{-1}, n^{\prime-1}, k n^{-1}$ and $N^{-1}$ in the expression of $\operatorname{MSE}(t(j))$. Here $t(1)=t_{T R 1}, t(2)=t_{T P 1}$, $t(3)=t_{T R 2}$ and $t(4)=t_{T P 2}$.

Let us define a function $\phi$ for obtaining the optimum values of $n^{\prime}, n, k$ and for minimizing the $\operatorname{MSE}(t(j))$ in case of fixed cost $C \leq C_{0}$ which is given as 
$\phi=\operatorname{MSE}(t(j))+\lambda_{j}\left\{c_{1}^{\prime} n^{\prime}+n\left(c_{1}+c_{2} W_{1}+c_{3} \frac{W_{2}}{k}\right)-C_{0}\right\}$

where $\lambda_{j}$ is Lagrange's multiplier.

By differentiating $\phi$ with respect to $n^{\prime}, n, k$ and equating to zero, we get the optimum values of $n^{\prime}, n$ and $k$ in case of fixed cost $C \leq C_{0}$ which are given as follows:

$$
\begin{aligned}
& n^{\prime}=\sqrt{\frac{V_{1 j}}{\lambda_{j} c_{1}^{\prime}}}, \\
& n=\sqrt{\frac{\left(V_{0 j}+k V_{2 j}\right)}{\lambda_{j}\left(c_{1}+c_{2} W_{1}+c_{3} \frac{W_{2}}{k}\right)}}
\end{aligned}
$$

and

$$
k_{o p t}=\sqrt{\frac{c_{3} W_{2} V_{0 j}}{\left(c_{1}+c_{2} W_{1}\right) V_{2 j}}},
$$

where

$$
\sqrt{\lambda_{j}}=\frac{1}{C_{0}}\left[\sqrt{V_{1 j} c_{1}^{\prime}}+\sqrt{\left(V_{0 j}+k_{o p t} V_{2 j}\right)\left(c_{1}+c_{2} W_{1}+c_{3} \frac{W_{2}}{k_{o p t}}\right)}\right]
$$

By putting the optimum values of $n^{\prime}, n$ and $k$ from (5.5), (5.6) and (5.7) in equation (5.3) and neglecting the term of $\operatorname{order}(1 / N)$, we get the minimum mean square error of $t(j)$ in case of the fixed cost $C \leq C_{0}$ which is given as:

$$
\begin{aligned}
& \operatorname{MSE}(t(j))_{\min }=\left[\frac { 1 } { C _ { 0 } } \left\{\sqrt{V_{1 j} c_{1}^{\prime}}+\right.\right. \\
& \left.\left.\left(V_{0 j}+k_{o p t} V_{2 j}\right)\left(c_{1}+c_{2} W_{1}+c_{3} \frac{W_{2}}{k_{o p t}}\right)\right\}^{2}\right]
\end{aligned}
$$

\section{DETERMINATION OF $n^{\prime}, n$ AND $k$ FOR THE SPECIFIED VARIANCE $V=V_{0}$}

Let $V_{0}$ be the specified variance of the estimator $t(j)$ which is fixed in advance. So we have

$V_{0}=\frac{1}{n} V_{0 j}+\frac{1}{n^{\prime}} V_{1 j}+\frac{k}{n} V_{2 j}-\frac{1}{N} V_{3 j}$,

For minimizing the average total cost $C$ for the specified variance (i.e. $\left.\operatorname{MSE}(t(j))=V_{0}\right)$ of the estimator $t(j)$ and for obtaining the optimum values of $n^{\prime}, n$ and $k$, we define a function $\psi$ which is given as:

$\psi=\left\{c_{1}^{\prime} n^{\prime}+n\left(c_{1}+c_{2} W_{1}+c_{3} \frac{W_{2}}{k}\right)\right\}+\mu_{j}\left(\operatorname{MSE}(t(j))-V_{0}\right)$,

where $\mu_{j}$ is Lagrange's multiplier.

By differentiating $\psi$ with respect to $n^{\prime}, n, k$ and equating to zero, we get the optimum values of $n^{\prime}, n$ and $k$ in case of specified variance which are given as follows:

$$
\begin{aligned}
& n^{\prime}=\sqrt{\frac{\mu_{j} V_{1 j}}{c_{1}^{\prime}}}, \\
& n=\sqrt{\frac{\mu_{j}\left(V_{0 j}+k V_{2 j}\right)}{\left(c_{1}+c_{2} W_{1}+c_{3} \frac{W_{2}}{k}\right)}}
\end{aligned}
$$

and

$$
k_{o p t}=\sqrt{\frac{c_{3} W_{2} V_{0 j}}{\left(c_{1}+c_{2} W_{1}\right) V_{2 j}}},
$$

where

$$
\sqrt{\mu_{j}}=\frac{\left[\sqrt{c_{1}^{\prime} V_{1 j}}+\sqrt{\left(V_{0 j}+k_{o p t} V_{2 j}\right)\left(c_{1}+c_{2} W_{1}+c_{3} \frac{W_{2}}{k_{o p t}}\right)}\right]}{\left[V_{0}+\frac{1}{N} V_{3 j}\right]}
$$

By putting the optimum values of $n^{\prime}, n$ and $k$ from (6.3), (6.4) and (6.5) in equation (5.2) and neglecting the term of $\operatorname{order}(1 / N)$, we get the minimum expected total cost of the estimator $t(j)$ for the specified variance $V=V_{0}$ which is given as follows:

$C(t(j))_{\min }=\frac{\left[\sqrt{c_{1}^{\prime} V_{1 j}}+\sqrt{\left(V_{0 j}+k_{o p t} V_{2 j}\right)\left(c_{1}+c_{2} W_{1}+c_{3} \frac{W_{2}}{k_{o p t}}\right)}\right]^{2}}{V_{0}}$

\section{EMPIRICAL STUDIES \\ VII.1 Data Set I [Khare and Sinha [24]]}

The present data belong to the data on growth of upper socio- economic group of 95 school going children of Varanasi under an ICMR study, Department of Pediatrics, 
BHU during 1983-84 has been taken under study. The first $25 \%$ (i.e. 24 children) units have been considered as nonresponse units. The values of parameters related to the study characters $y_{1}$ (the height of the children in $\mathrm{cm}$ ) and $y_{2}$ (weight of the children in $\mathrm{kg}$ ) and auxiliary character $x$ (chest circumference of the children in $\mathrm{cm}$ ) have been given as follows:

$\bar{Y}_{1}=115.9526, \quad \bar{Y}_{2}=19.4968, \quad \bar{X}=55.8611$, $C_{y 1}=0.0515, \quad C_{y 2}=0.1561, \quad C_{x}=0.0586$,
$C_{y 1}^{\prime}=0.0440, \quad C_{y 2}^{\prime}=0.1208, \quad C_{x}^{\prime}=0.0540$,

$\rho_{y 1 x}=0.620, \quad \rho_{y 2 x}=0.846, \quad \rho_{y 1 y 2}=0.713$,

$\rho_{y 1 x}^{\prime}=0.401, \quad \rho_{y 2 x}^{\prime}=0.729, \quad \rho_{y 1 y 2}^{\prime}=0.678$

The problem considered is to estimate the ratio between height and weight of the male children aged 6-7 years using chest circumference as the auxiliary character.

Table 1. Relative efficiency (RE) and mean square error (MSE) of the proposed estimators with respect to $\hat{R}\left(N=95, n^{\prime}=65, n=30\right)$

\begin{tabular}{|c|c|c|c|c|c|c|}
\hline \multirow{2}{*}{ Estimators } & \multicolumn{7}{|c|}{$1 / k$} & \multicolumn{2}{c|}{$1 / 2$} \\
\cline { 2 - 7 } & \multicolumn{2}{|c|}{$1 / 4$} & \multicolumn{2}{c|}{$1 / 3$} & RE & MSE \\
\cline { 2 - 7 } & $\mathrm{RE}$ & $\mathrm{MSE}$ & $\mathrm{RE}$ & $\mathrm{MSE}$ & $\mathrm{R}$ & \multicolumn{7}{c|}{0.01533} \\
\hline$\hat{R}$ & 100.00 & 0.02088 & 100.00 & 0.01810 & 100.00 & 0.01123 \\
\hline$t_{R 1}$ & 139.64 & 0.01495 & 138.27 & 0.01309 & 136.46 & 0.01123 \\
\hline$t_{R 2}$ & 117.98 & 0.01769 & 121.32 & 0.01492 & 126.19 & 0.01215 \\
\hline
\end{tabular}

Table 2. Relative efficiency (RE) and mean square error (MSE) of the estimators with respect to $\hat{R}$ for the fixed cost $\mathrm{C}_{0}=$ Rs. 250

\begin{tabular}{|c|c|c|c|c|c|}
\hline \multirow{2}{*}{ Estimators } & \multicolumn{4}{|c|}{$c_{1}^{\prime}=R s .0 .50, \quad c_{1}=R s .5, \quad c_{2}=R s .15, \quad c_{3}=R s .25$} \\
\cline { 2 - 6 } & $k_{\text {opt }}$ & $\begin{array}{c}n_{\text {opt }}^{\prime} \\
\text { (approx.) }\end{array}$ & $\begin{array}{c}n_{\text {opt }} \\
\text { (approx.) }\end{array}$ & $\begin{array}{c}\text { RE } \\
\text { (in \%) }\end{array}$ & MSE \\
\hline$\hat{R}$ & 1.48 & --- & 12 & 100.00 & 0.0483 \\
\hline$t_{R 1}$ & 1.35 & 50 & 11 & 138.56 & 0.0349 \\
\hline$t_{R 2}$ & 1.16 & 47 & 10 & 118.75 & 0.0407 \\
\hline
\end{tabular}

Table 3. Expected cost (in Rs.) of the estimators for the specified variance $V_{0}=0.0561$

\begin{tabular}{|c|c|c|c|c|}
\hline \multirow{2}{*}{ Estimators } & \multicolumn{2}{|c|}{$c_{1}^{\prime}=R s .0 .50, \quad c_{1}=R s .5, \quad c_{2}=R s .15, \quad c_{3}=R s .25$} \\
\cline { 2 - 5 } & $k_{\text {opt }}$ & $\begin{array}{c}n_{\text {opt }}^{\prime} \\
\text { (approx.) }\end{array}$ & $\begin{array}{c}n_{\text {opt }} \\
\text { (approx.) }\end{array}$ & $\begin{array}{c}\text { Expected cost } \\
\text { (in Rs.) }\end{array}$ \\
\hline$\hat{R}$ & 1.48 & --- & 11 & 215.43 \\
\hline$t_{R 1}$ & 1.35 & 31 & 7 & 155.48 \\
\hline$t_{R 2}$ & 1.16 & 34 & 8 & 181.41 \\
\hline
\end{tabular}


From table 1, It has been observed that for the fixed values of $N, n^{\prime}, n$ and different values of $k$, the proposed estimator's $t_{R 1}$ and $t_{R 2}$ are more efficient in comparison to the usual estimator $\hat{R}$. The estimator $t_{R 1}$ is more efficient in comparison to the estimator $t_{R 2}$ for different values of $k$. The values of MSE of all estimators decrease as the values of $k$ decrease.

From table 2, it has been observed that for the fixed cost, the proposed estimators $\left(t_{R 1}, t_{R 2}\right)$ have less MSE in comparison to the usual estimator $\hat{R}$ and $t_{R 1}$ have less MSE in comparison to the estimator $t_{R 2}$.

From table 3 , it has been observed that for the specified variance $V_{0}$, the proposed estimators $\left(t_{R 1}, t_{R 2}\right)$ have less total cost in comparision to the relevant estimator $\hat{R}$.

\section{VII.2 Data Set II [Sinha [28]]}

109 Village/Town/Ward wise population of urban area under Police-station-Baria, Tahasil Champua, Orissa has been taken under consideration from District Census Handbook, 1981, Orissa, published by Govt. of India. The last $25 \%$ villages (i.e. 27 villages) have been considered as nonresponse group of the population. Here we have take the study characters and auxiliary character as follows:

$y_{1}$ - Number of occupied residential house in the village.

$y_{2}$ - Average number of person in a house in the village.

$x$ - Number of Cultivator's in the village.

The values of the parameters of the population under study are given as follows:

$$
\begin{array}{lcc}
\bar{Y}_{1}=88.3670, & \bar{Y}_{2}=5.5832, & \bar{X}=100.5505, \\
C_{y 1}=0.6713, & C_{y 2}=0.1079, & C_{x}=0.7314, \\
C_{y 1}^{\prime}=0.5123, & C_{y 2}^{\prime}=0.0900, & C_{x}^{\prime}=0.5678, \\
\rho_{y 1 x}=0.795, & \rho_{y 2 x}=-0.084, & \rho_{y 1 y 2}=-0.194, \\
\rho_{y 1 x}^{\prime}=0.658, & \rho_{y 2 x}^{\prime}=0.092, & \rho_{y 1 y 2}^{\prime}=0.023
\end{array}
$$

\begin{tabular}{|c|c|c|c|c|c|c|}
\hline \multirow[b]{3}{*}{ Estimators } & \multicolumn{6}{|c|}{$1 / k$} \\
\hline & \multicolumn{2}{|c|}{$1 / 4$} & \multicolumn{2}{|c|}{$1 / 3$} & \multicolumn{2}{|c|}{$1 / 2$} \\
\hline & RE & MSE & RE & MSE & RE & MSE \\
\hline$\hat{P}$ & 100.00 & 2905.62 & 100.00 & 2494.60 & 100.00 & 2083.58 \\
\hline$t_{P 1}$ & 167.82 & 1731.37 & 166.92 & 1494.46 & 165.69 & 1257.54 \\
\hline$t_{P 2}$ & 128.93 & 2253.69 & 135.38 & 1842.67 & 145.54 & 1431.65 \\
\hline
\end{tabular}

Table 4. Relative efficiency (RE) and mean square error (MSE) of the proposed estimators with respect to $\hat{P}\left(N=109, n^{\prime}=70, n=40\right)$

Table 5. Relative efficiency (RE) and mean square error (MSE) of the estimators with respect to $\hat{P}$ for the fixed cost $\mathrm{C}_{0}=$ Rs. 250

\begin{tabular}{|c|c|c|c|c|c|}
\hline \multirow{2}{*}{ Estimators } & \multicolumn{2}{|c|}{$c_{1}^{\prime}=R s .0 .50, \quad c_{1}=R s .3, \quad c_{2}=R s .10, \quad c_{3}=R s .30$} \\
\cline { 2 - 6 } & $k_{\text {opt }}$ & $\begin{array}{c}n_{\text {opt }}^{\prime} \\
\text { (approx.) }\end{array}$ & $\begin{array}{c}n_{\text {opt }} \\
\text { (approx.) }\end{array}$ & $\begin{array}{c}\text { RE } \\
\text { (in \%) }\end{array}$ & \multicolumn{2}{c|}{ MSE } \\
\hline$\hat{P}$ & 1.96 & --- & 17 & 100.00 & 6955.03 \\
\hline$t_{P 1}$ & 1.62 & 83 & 14 & 157.77 & 4408.44 \\
\hline$t_{P 2}$ & 1.10 & 81 & 12 & 151.69 & 4584.92 \\
\hline
\end{tabular}


Table 6. Expected cost (in Rs.) of the estimators for the specified variance $V_{0}=4550$

\begin{tabular}{|c|c|c|c|c|}
\hline \multirow{2}{*}{ Estimators } & \multicolumn{2}{|c|}{$c_{1}^{\prime}=R s .0 .50, \quad c_{1}=R s .3, \quad c_{2}=R s .10, \quad c_{3}=R s .30$} \\
\cline { 2 - 5 } & $k_{\text {opt }}$ & $\begin{array}{c}n_{\text {opt }}^{\prime} \\
\text { (approx.) }\end{array}$ & $\begin{array}{c}n_{\text {opt }} \\
\text { (approx.) }\end{array}$ & $\begin{array}{c}\text { Expected cost } \\
\text { (in Rs.) }\end{array}$ \\
\hline$\hat{P}$ & 1.96 & --- & 27 & 382.14 \\
\hline$t_{P 1}$ & 1.62 & 80 & 13 & 242.22 \\
\hline$t_{P 2}$ & 1.10 & 82 & 12 & 251.92 \\
\hline
\end{tabular}

From table 4, It has been observed that for the fixed values of $N, n^{\prime}, n$ and different values of $k$, the proposed estimator's $t_{P 1}$ and $t_{P 2}$ are more efficient in comparison to the usual estimator $\hat{P}$. The estimator $t_{P 1}$ is more efficient in comparison to the estimator $t_{P 2}$ for different values of $k$. The values of mean square error of all estimators decrease as the values of $k$ decrease.

From table 5, it has been observed that for the fixed cost, the proposed estimators $\left(t_{P 1}, t_{P 2}\right)$ have less MSE in comparison to the usual estimator $\hat{\boldsymbol{P}}$ and $t_{P 1}$ have less MSE in comparison to the estimator $t_{P 2}$.

From table 6 , it has been observed that for the specified variance $V_{0}$, the proposed estimators $\left(t_{P 1}, t_{P 2}\right)$ have less total cost in comparison to the usual estimator $\hat{P}$ and $t_{P 1}$ have less total cost in comparison to the estimator $t_{P 2}$.

\section{CONCLUSION}

From tables 1, 2, 3, we conclude that when linear relationship between study character and auxiliary character is not very strong, the proposed estimators for ratio of two population means in the presence of non-response are more efficient in comprision to the usual estimator in case of the fixed sample sizes and also in case of the fixed cost. The proposed estimators have less total cost in comprision to the usual estimator in case of the specified variance.

From tables 4, 5, 6, we conclude that when linear relationship between study character and auxiliary character is not very strong, the proposed estimators for product of two population means in the presence of non-response are more efficient in comprision to the usual estimator in case of the fixed sample sizes and also in case of the fixed cost. The proposed estimators have less total cost in comprision to the usual estimator in case of the specified variance.

\section{ACKNOWLEDGMENT}

The authors are very much indebted to the editor-in-chief Dr. Chandra K Jaggi and learned referees for their valuable suggestions leading to improvement of the quality of contents and presentation of the original manuscript.

\section{REFERENCES}

[1] M.P. Singh, "On the estimation of ratio and product of population parameters", Sankhya B, 27, 321-328, 1965.

[2] M.P. Singh, "Ratio cum product method of estimation", Metrika, 12, 34-43, 1967.

[3] M.P. Singh, "Comparison of some ratio cum product estimators", Sankhya B, 31, 375-378, 1969.

[4] S.M. Shah, D.N. Shah, "Ratio cum product estimator for estimation ratio (product) of two population parameters", Sankhya C, 40,156-166, 1978.

[5] T.P. Tripathi, "A general class of estimators of population ratio", Sankhya C, 42, 63-75,1980.

[6] R.K. Singh, "On estimating ratio and product of population parameters", Calcutta Statist. Assoc. Bull., 20, 39-49,1982.

[7] H.P. Singh, "On the estimation of ratio and product of two finite populations means", Proc. Nat. Acad. Sci. India. Sec. A, 58, 399402,1998.

[8] R.S. Biradar, H.P. Singh, "A class of estimators for population parameters using supplementary information”, Aligarh J. Statist., $17 \& 18,54-71,1997-98$.

[9] L.N. Upadhyaya, G.N. Singh, H.P. Singh, "Use of transformed auxiliary variable in the estimation of population ratio in sample survey", Statistics in Transition, 4, 1019-1027, 2000.

[10] M.H. Hansen, W.N. Hurwitz, "The problem of non response in sample surveys", J. Amer. Statis. Assoc., 41, 517-529, 1946.

[11] P.S.R.S. Rao, "Ratio estimation with sub sampling the nonrespondents", Survey Methodology, 12, 217-230, 1986.

[12] P.S.R.S. Rao, "Ratio and regression estimates with sub sampling the non- respondents", Paper presented at a special contributed session of the International Statistical Association Meeting, Sept. 2-16, Tokyo, Japan,1987.

[13] B.B. Khare, S. Srivastava, "Transformed product type estimators for population mean in presence of soft-core observations", Proc. Math. Soci. B.H.U., 12, 29-34, 1996.

[14] B.B. Khare, S. Srivastava, "Transformed ratio type estimators for the population mean in the presence of non-response", Communi. Stat.-Theory and Methods, 26, 1779-1791,1997. 
[15] R. Singh, M. Kumar, M.K. Chaudhary, F. Smarandache, "Estimation of population mean in presence of non-response using exponential estimator", Paper published in the Book Multispace \& Multistructure Neutrosophic Transdisciplinarity 4, 758-768, 2010.

[16] S. Kumar, S. Bhougal, "Estimation of the population mean in presence of non-response", Communi. Kore. Stat. Soci., 18, 537$548,2011$.

[17] K. Kumar, M. Kumar, "Improved exponential ratio and product type estimators for population mean in the presence of nonresponse", Bullen. Math. Stats. Rese., 5, 68-76, 2017.

[18] B.B. Khare, S. Srivastava, "Estimation of population mean using auxiliary character in presence of non-response", The National Academy of Sciences, Letters, India, 16, 111-114,1993.

[19] B.B. Khare, S. Srivastava, "Study of conventional and alternative two phase sampling ratio, product and regression estimators in presence of non-response", Proceedings of the National Academy of Sciences, India Section A: Physical Sciences India, 65 (A) II, 195-203, 1995.

[20] H.P. Singh, S. Kumar, "Estimation of mean in presence of nonresponse using two phase sampling scheme", Statistical Papers, 50, 559-582, 2010.

[21] B.B. Khare, U. Srivastava, K. Kumar, “ An improved class of estimators for population mean using auxiliary character in the presence of non-response", The National Academy Science Letters, India, 35, 361-366, 2012.

[22] B.B. Khare, S.K. Pandey, " A class of estimators for ratio of two population means using auxiliary character in presence of nonresponse”, J. Sc. Res. B.H.U., 50, 115-124, 2000.

[23] B.B. Khare, R.R. Sinha, "Estimation of the ratio of two population means using auxiliary character with unknown population mean in presence of no-response", Prog. Maths. B.H.U., 36, 337-348, 2002.

[24] B.B. Khare, R.R.Sinha, "Estimation of the ratio of the two populations means using multi-auxiliary characters in the presence of non-response", Published in the Book Statistical Techniques in Life Testing, Reliability, Sampling Theory and Quality Control, 163-171, 2007.

[25] H.P. Singh, G.K. Vishwakarma, “ Modified exponential ratio and product estimators for finite population mean in double sampling", Austrian Journal of Statistics, 36(3), 217-225, 2007.

[26] R. Tailor, S. Chouhan, J.M. Kim, "Ratio and product type exponential estimators of population mean in double sampling for stratification”, Comm. Statist. Appl. \& Meth., 21(1), 1-9, 2014.

[27] A. Lakhre, "Dual to ratio and product type exponential estimators of finite population mean in double sampling for stratification", Inter. Jour. Sci. Res. Math. Stats. Sci., 4(5), 1-8, 2017.

[28] R.R. Sinha, "Some problems on the estimation of population parameters using multi auxiliary characters in presence of nonresponse", $\mathrm{PhD}$. Thesis submitted to the Banaras Hindu University, Varanasi, India, 2001.

\section{AUTHORS PROFILE}

Dr. Kamlesh Kumar has completed his Ph.D. (Statistics) in year 2012 from Department of Statistics, Banaras Hindu University, Varanasi, India. He is currently working as Assistant Professor (Biostatistics) in Department of Community Medicine, Rajkiya Medical College, Jalaun (Orai), U.P., India. He has published more than 12 research papers in reputed International and National Journals and also presented 6 research papers in International and National Conferences.
Dr. Mukesh Kumar joined as Assistant Professor in September 2013 in Department of Statistics, Central university of South Bihar, Bihar, India. He has completed his $\mathrm{PhD}$ degree in year 2011 from Department of Statistics, Banaras Hindu University, Varanasi, India. He has awarded JRF and SRF during the PhD from UGC under the fellowship "UGC Research Fellowship in Sciences for Meritorious Students" (RFSMS). He has published 15 research papers in International / National Journals and Book chapters. 